\title{
Anisotropic surface energy formulations and their effect on stability of a growing thin film
}

\author{
MACIEK D. KORZEC
}

Department of Mathematics, Technische Universität Berlin, Straße des 17. Juni 136, 10623 Berlin, Germany

E-mail:korzec@math.tu-berlin.de

ANDREAS MÜNCH

Mathematical Institute, University of Oxford, 24-29 St Giles', Oxford, OX1 3LB, UK

E-mail: muench@maths.ox.ac.uk

\section{BARBARA WAGNER}

Department of Mathematics, Technische Universität Berlin, Straße des 17. Juni 136, 10623 Berlin, Germany

E-mail: bwagner@math.tu-berlin.de

[Received 29 December 2011]

\begin{abstract}
In this paper we revisit models for the description of the evolution of crystalline films with anisotropic surface energies. We prove equivalences of symmetry properties of anisotropic surface energy models commonly used in the literature. Then we systematically develop a framework for the derivation of surface diffusion models for the self-assembly of quantum dots during Stranski-Krastanov growth that include surface energies also with large anisotropy as well as the effect of wetting energy, elastic energy and a randomly perturbed atomic deposition flux.

A linear stability analysis for the resulting sixth-order semilinear evolution equation for the thin film surface shows that that the new model allows for large anisotropy and gives rise to the formation of anisotropic quantum dots. The nonlinear three-dimensional evolution is investigated via numerical solutions. These suggest that increasing anisotropy stabilizes the faceted surfaces and may lead to a dramatic slow-down of the coarsening of the dots.
\end{abstract}

2010 Mathematics Subject Classification: Primary 35G20; Secondary 74S25.

Keywords: Anisotropic surface energy, high order partial differential equations, pseudospectral methods, surface diffusion.

\section{Introduction}

Modeling, analysis and numerical simulation of the self-assembly of thin nanostructured crystalline films is of enormous interest for many emerging applications in the semiconductor industries, for the synthesis of multifunctional materials or in the design of next-generation solar cells. A fundamental understanding of the underlying principles that control the formation of patterns as well as their evolution, forms the basis for optimizing the properties of new materials, such as e.g. the positioning, size and shape of superlattices of quantum dots to increase of conversion efficiencies of thin-film solar cells, see e.g. [3].

One of the most prominent examples for the patterning of surfaces is the quantum dot selfarrangement during epitaxial growth which has been studied in numerous works. In particular, for 
heteroepitaxial growth of the systems $\mathrm{Ge} / \mathrm{Si}$ and $\mathrm{SiGe} / \mathrm{Si}$ there exists a sizable body of literature that is concerned with a precise understanding of the processes on the nanoscale. The review by Drucker [12] and many of the references therein yield a detailed overview of the experimental works.

In order to capture and predict the long-time evolution of large scale three-dimensional nanostructures continuum models have shown to be very useful. They involve the coupling of the elasticity problems for the film and the underlying substrate with the evolution of the film surface $h(x, y, t)$ based on Mullins' surface diffusion equation [31, 39]

$$
h_{t}=\sqrt{1+|\nabla h|^{2}} \ggg \nabla_{s}^{2} \mu
$$

where $|\cdot|$ is the Euclidean norm, $\nabla_{s}^{2}$ is the surface Laplacian, see e.g. [44], $D$ is a diffusion constant and

$$
\mu=\varepsilon_{\text {sed }}+\varepsilon_{\text {surf }}
$$

the chemical potential with contributions arising from the strain energy

$$
\varepsilon_{\text {sed }}=1 /\left.2 \sum_{i, j} \sigma_{i j} \epsilon_{i j}\right|_{z=h}
$$

evaluated at the surface, with the strain and stress tensor, $\left(\epsilon_{i j}\right)_{i j}$ and $\left(\sigma_{i j}\right)_{i j}$, respectively, and the surface free energy $\varepsilon_{\text {surf }}$. The derivation of the latter will be discussed in detail in this study.

For the resulting free boundary problems, asymptotic methods leading via a small-slope reduction of the system to a single high-order evolution equation for the film profile, have previously been developed, see in particular the works by Spencer et al. [37, 39]. Extensions of these studies that in addition account for effects of substrate-film wetting or anisotropy, see, e.g., $[17,24,44]$, show that for such reduced models large-scale numerical simulations of the formation of nanostructures are feasible and can also be evolved on a long time-scale to yield information on their coarsening behavior.

Further extensions of this approach are important for the implementations of superlattices, such as for example the $\mathrm{SiGe/Si}$ system as has been studied by Sutter et al. [42] or the similar $\mathrm{Ge} / \mathrm{Si}$ system investigated by Chen et al. [6] to design and optimize material systems relevant for photovoltaic applications, see also [20,21] and the review by Springholz and Holy [41]. To make progress using above approach, a thorough understanding of the important physical effects and how to incorporate them systematically into a realistic model, even for the basic one-layer heteroepitaxial growth on a substrate, is crucial.

In the first part of this study (Section 2) we first revisit the different formulations for surface energy. In a purely surface energy driven system the chemical potential can be derived from the functional derivative of the surface free energy via the surface integral over the surface energy density $\gamma$;

$$
\varepsilon_{\text {surf }}=\frac{\delta}{\delta h} \int \gamma d S .
$$

We discuss different formulations for $\gamma$ that have been used in literature and show equivalence of symmetries. In particular, we show how the standard cubic symmetry properties of a surface energy $\gamma(n)$, expressed in terms of the normal $n$ pointing out of the film, relate to the gradient formulation $\gamma\left(h_{x}, h_{y}\right)$, which is used for the derivation of the thin film evolution equation. We 
systematically develop a framework that incorporates the specific properties of the film-substrate system and naturally extends to the case that includes strong anisotropy.

The analysis and results are used in the following Section 3 for the derivation of a new thin-film equation and leads, via asymptotic long-wave approximations, to a sixth-order semi-linear equation for $h(x, y, t)$ that accounts for an elastic field in the film and in the substrate, wetting layer, strong surface energy anisotropy in the film and a randomly perturbed atomic flux, extending the models by Tekalign and Spencer [44] and Savina et al. [34].

In the final Section 4 we investigate the linear stability results for increasing anisotropy of the surface energy and compare them to our numerical solution of the evolving patterns of the two-dimensional surface $h(x, y, t)$. For the numerical scheme used to approximate the solutions of the sixth-order semi-linear PDE we employ pseudo-spectral methods using exponential time differencing (ETD) [11, 22], and a third order semi-implicit backward differentiation (SBDF) scheme.

We discuss the impact of the corner energy on the faceting that appears as a sixth-order Wilmore regularization in the model equation. We find that large anisotropy coefficients lead to stabilized faceted arrays and a slow-down of the coarsening process.

\section{Surface energy density formulations}

First we revisit the different surface energy formulations that have been used in the literature for growing surfaces, discuss equivalence of symmetry characteristics of the surface energies, followed by the derivation of general expressions for the constituent terms for the chemical potential.

The first continuum models for epitaxial growth were actually rather concerned with the elastic problem than the surface energy, neither intermolecular interactions between film and substrate nor anisotropy have been considered, see [37, 39, 40]. The surface energy was assumed to be isotropic together with surface diffusion and stress fields [38, 47, 49, 50]. We denote this class of surface energy models by $\mathbf{S E}_{I}: \gamma=$ const. Consequently neither wetting layer connecting the nanostructures nor faceting of the growing dots could be captured although this is a signature of the Stranski-Krastanov growth mode.

Golovin et al. [17] extended the theory by including wetting effects. In later works such as by Pang and Huang [32, 33] or Tekalign and Spencer [44, 45] such an extension has been incorporated by letting $\gamma$ depend not only on the gradients of the surface profile to model the anisotropy, but also on the surface height $h$ itself. This dependency has been be modeled in the framework of the boundary layer theory by Spencer [35]. It constituted a fundamental result for modeling StranskiKrastanov systems and was followed by many further studies, e.g. [1, 5, 17, 32, 44, 45]. We denote this class here by $\mathbf{S E}_{I I}: \gamma=\gamma(h)$.

Since crystalline materials are inherently anisotropic as a result of the regular structure defined by their bravais lattices, preferred orientations corresponding to lower free energy states emerge during growth. For the heteroepitaxial case, cusped anisotropic surface energies have been proposed by Eisenberg and Kandel [13-15], while combinations of wetting and anisotropic surface energies were considered by Chiu and Huang $[9,10]$. For these situations the family of $\mathrm{SE}_{I I}$ can be extended by letting the surface energy density also be a function of the gradients of the surface, denoted here by $\mathbf{S E}_{I I I}: \gamma=\gamma\left(h, h_{x}, h_{y}\right)$.

Often the surface anisotropy is expressed in terms of angles or the outward unit normal as arguments, and as Figure 1 (c) indicates for the one-dimensional case, the formulations are 


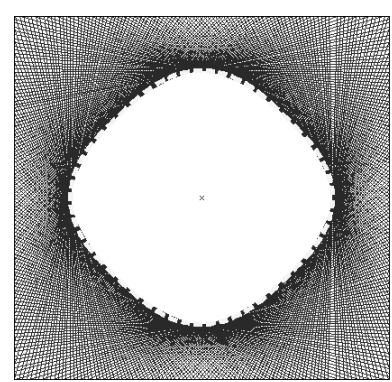

(a)

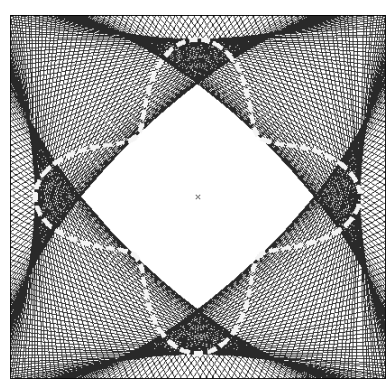

(b)

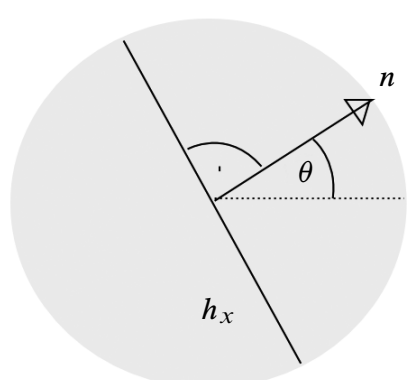

(c)

FIG. 1. Polar plots (dashed lines) of the surface energy $\gamma(\theta)=1+G \cos (4 \theta)$ and the corresponding equilibrium shapes (white areas) constructed with the Wulff construction for (a), the weak anisotropy strength $G=0.05$, and (b) the strong anisotropy $G=\mathbf{0 . 3}$. The latter leads to "ears" in the Wulff construction and corners in the equilibrium shape. (c) shows the orientation dependency in one dimension expressed in terms of the slope $h_{x}$, the outward unit normal $n$ or the facet angle $\theta$.

equivalent as long as the gradients of the surface stay away from infinity. We will discuss these relations in more detail in the following paragraphs.

This family of surface energies has been shown to be very useful for the description of a StranskiKrastanov systems in a long-wave approximation, see e.g. [7-10, 24, 48]. However, all of these models will eventually become ill-posed for large enough anisotropy of the surface energy. This corresponds to the appearance of sharp edges in the film surface, as can be seen in Figure 1, where the Wulff construction is applied to obtain the equilibrium shapes (see [46] or [19]). In (a) the anisotropy is weak, which means that the polar plot of $\gamma(\theta)=1+G \cos (4 \theta)$ (dashed line) is convex for $G=0.05$ and it yields a smooth shape from the Wulff construction (white area). In (b) the anisotropy strength is increased to $G=0.3$ and while the polar plot becomes nonconvex, the Wulff construction shows distinct "ears" and the equilibrium shape has sharp corners.

An extension of the surface energy that includes higher order gradients is called Wilmore regularization. It was introduced by Golovin et al. [16] in connection with crystallization of Si from a melt. Further studies that are mainly concerned with equilibrium shapes e.g. Spencer [36], analyze the influence of the corner regularization onto the stationary geometry by using matched asymptotic expansions. Savina et al. [34] proposed a model for the faceting of growing surfaces in the absence of elastic stresses that used this regularization. The model was further analyzed with respect to its stationary states and existence of unique solutions in [25-27] and it has been extended by Golovin et al. [18] who also considered wetting interactions in the anisotropic surface energy. Both works are not related to growth based on the Asaro-Tiller-Grinfeld instability since no elastic subproblem resulting from lattice mismatch is considered.

The dependence of the surface energy on higher order gradients has further been addressed by several other works in crystal growth theory, such as in $[4,34,36]$. In what follows, we denote this family by $\mathbf{S E}_{I V}: \gamma=\gamma\left(h, h_{x}, h_{y}, h_{x x}, h_{x y}, h_{y y}\right)$. In particular this includes models of the type $\gamma=\gamma(n, \kappa)$, where $n$ is the outward unit normal and $\kappa$ is the mean curvature of the growing film.

\subsection{Symmetry and surface energy properties}

We show the equivalences of the different formulations for the surface energy and begin by proving how cubic symmetry relates an outward unit normal description to an angle parametrization. 
Secondly we show in Proposition 2.3 that there is an equivalent formulation in terms of the dependency on the surface gradients when $h$ is a regularly growing surface. Finally, we show how to incorporate such surface energies in a surface diffusion equation.

The spatial derivatives of the evolving surface, $h_{x}$ and $h_{y}$, serve as arguments for the families of surface energies $\mathrm{SE}_{I I I}$ and $\mathrm{SE}_{I V}$. This dependency is used to model the anisotropy of the surface energy. Typically, the outward unit normal $n=\left(n_{1}, n_{2}, n_{3}\right)$ is used as argument for $\gamma$. Then for crystals with e.g. cubic symmetry the regular structure implies

$$
\gamma_{1}\left(n_{1}, n_{2}, n_{3}\right)=\gamma_{1}\left(\pi\left(n_{1}, n_{2}, n_{3}\right)\right)=\gamma_{1}\left(\delta_{1} n_{1}, \delta_{2} n_{2}, \delta_{3} n_{3}\right), \quad \delta_{i} \in\{ \pm 1\},
$$

for any permutation $\pi$ of the three components, see, e.g., McFadden [30]. It is a natural idea to believe that these properties have an analogon in terms of a polar description of the unit normal. In polar coordinates $\theta_{1}, \theta_{2}$ the symmetry property

$$
\gamma_{2}\left(\theta_{1}, \theta_{2}\right)=\gamma_{2}\left(\theta_{1} \pm \pi / 2, \theta_{2} \pm \pi / 2\right)=\gamma_{2}\left(\delta_{1} \theta_{1}, \delta_{2} \theta_{2}\right), \quad \delta_{i} \in\{ \pm 1\} .
$$

appears natural. In the one-dimensional case, for the square symmetry $\gamma(\theta)=1+G \cos (4 \theta)$, one can easily check that the above statement holds (reduced by one dimension). However, we will show that the symmetry properties of $\gamma_{1}$ and $\gamma_{2}$ are not equivalent and that (2.2) follows from (2.1), but not vice versa.

For regular surfaces, where the outward unit normal is a function of $h_{x}$ and $h_{y}$, the surface energy can be written as $\gamma_{3}\left(h_{x}, h_{y}\right)=\gamma_{1}\left(n\left(h_{x}, h_{y}\right)\right)$, then the symmetry defined by (2.1) transforms to

$$
\gamma_{3}\left(h_{x}, h_{y}\right)=\gamma_{3}\left(h_{y}, h_{x}\right)=\gamma_{3}\left(\delta_{1} h_{x}, \delta_{2} h_{y}\right), \quad \delta_{i} \in\{ \pm 1\} .
$$

Symmetries in (2.1) and (2.3) are indeed equivalent for regular surfaces (those which are regularly parametrizable from the plane). However, we first discuss the symmetries (2.1) and (2.2), therefore we state the following lemma on description of symmetry (2.1) in terms of Householder reflections:

Lemma 2.1 Let $H\left(a_{j}\right)=I d-2 a_{j} a_{j}^{T}, j=1, \ldots, 5$, be Householder reflections with the normalized cubic symmetry axes

$$
\begin{aligned}
& a_{1}=e_{1}, a_{2}=e_{2}, a_{3}=e_{3}, \\
& a_{4}=(1,-1,0) / \sqrt{2}, a_{5}=(1,0,-1) / \sqrt{2},
\end{aligned}
$$

where $e_{j}, j=1,2,3$ are the three Cartesian basis vectors, then symmetry properties (2.1) are equivalent to $\gamma(n)=\gamma\left(H\left(a_{j}\right) n\right), j=1, \ldots, 5$.

The proofs of the lemma as well as the following propositions are given in the appendix. For the next proposition we let $n \in \mathbb{S}^{2}$ (the unit sphere in $\mathbb{R}^{3}$ ), then $n$ can be expressed as rotation of $e_{3}$, the third Cartesian basis vector, in the $(x, y)-$ and in $(x, z)$-planes defined by the angles $\theta_{1}$ and $\theta_{2}$ and obtain

PROPOSITION 2.2 For

$$
\gamma_{2}\left(\theta_{1}, \theta_{2}\right)=\gamma_{1}\left(\sin \left(\theta_{2}\right) \cos \left(\theta_{1}\right),-\sin \left(\theta_{1}\right) \cos \left(\theta_{2}\right), \cos \left(\theta_{2}\right)\right)
$$

(2.1) and (2.2) are not equivalent, but the implication $(2.1) \Rightarrow(2.2)$ is true. 
In Section 3 we will express the surface energy in terms of $\gamma_{3}$, hence it is important how this formula relates to the other formulations. Since the gradients of a regular surface $h$ are not infinite, the outward normal (or the angles $\theta_{j}$ ), cannot be chosen arbitrarily. The next proposition states the equivalence of (2.1) and (2.3) for such smooth surfaces.

PROPOSITION 2.3 Let $h: \Omega \rightarrow \mathbb{R},(x, y) \mapsto h(x, y)$ be the smooth parameterization of a connected surface $m$ over a bounded domain $\Omega \subset \mathbb{R}^{2}$. Furthermore, let $n: \mathbb{R}^{2} \rightarrow \mathbb{S}^{2},(a, b) \mapsto$ $(-a,-b, 1) / \sqrt{1+a^{2}+b^{2}}$ be the outward unit normal function which maps onto the unit sphere $\mathbb{S}^{2}$. Then by defining $\gamma_{3}: \mathbb{R}^{2} \rightarrow \mathbb{R}, \gamma_{3}:=\gamma_{1} \circ n$ the symmetries (2.1) and (2.2) are equivalent.

Similar relations can be derived for other symmetry groups, here we focus on the symmetry properties of diamond cubic semiconductor materials, such as silicon and germanium. In the following the anisotropic surface energy for small surface gradients will be expressed as

$$
\gamma\left(h_{x}, h_{y}\right)=\sum_{k=0}^{\infty} \sum_{j=0}^{k} g_{k j} h_{x}^{j} h_{y}^{k-j},
$$

where for practical use the first sum will be truncated to be finite. In general the anisotropy coefficients $g_{k j}$ are given constants, e.g. supplied by experimental data. The chemical potential part that stems from the surface energy (1.4) can now be derived for the four energy types $\mathrm{SE}_{k}, k \in\{I, I I, I I I, I V\}$. Under sufficient regularity assumptions the following result holds:

PRoposition $2.4\left(\mathrm{SE}_{I V}\right)$ The variational derivative of the surface free energy based on $\mathrm{SE}_{I V}$ leads to the four term expression

$$
\varepsilon_{\text {surf }}=\varepsilon_{\kappa}+\varepsilon_{w e t}+\varepsilon_{a n i s}+\varepsilon_{h o t}
$$

with

$$
\begin{aligned}
\varepsilon_{\kappa} & =-\gamma \kappa, \\
\varepsilon_{w e t} & =n_{3} \partial_{h} \gamma \\
\varepsilon_{\text {anis }} & =-2\left(\frac{h_{x} h_{x x}+h_{y} h_{x y}}{N} \partial_{h_{x}} \gamma+\frac{h_{y} h_{y y}+h_{x} h_{x y}}{N} \partial_{h_{y}} \gamma\right)-N \partial_{x} \partial_{h_{x}} \gamma-N \partial_{y} \partial_{h_{y}} \gamma, \\
\varepsilon_{\text {hot }} & =\nabla^{2} \nabla_{\Delta h}(\gamma N),
\end{aligned}
$$

where

$$
N=\sqrt{1+h_{x}^{2}+h_{y}^{2}}, \nabla_{\nabla h}=\left(\partial_{h_{x}}, \partial_{h_{y}}\right)^{T} \text { and } \nabla_{\Delta h}=\partial_{h_{x x}}+\partial_{h_{y y}},
$$

so that

$$
\nabla \cdot \nabla_{\nabla h}=\partial_{x} \partial_{h_{x}}+\partial_{y} \partial_{h_{y}} \text { and } \quad \nabla^{2} \nabla_{\Delta h}=\partial_{x x} \partial_{h_{x x}}+\partial_{x x} \partial_{h_{y y}}+\partial_{y y} \partial_{h_{x x}}+\partial_{y y} \partial_{h_{y y}}
$$


Proof. With the above notation one can easily check the following equalities:

$$
\begin{aligned}
\varepsilon_{\text {surf }}= & \frac{\delta}{\delta h} \int \gamma\left(h, h_{x}, h_{y}, h_{x x}, h_{x y}, h_{y y}\right) N d x d y \\
= & N \partial_{h} \gamma-\nabla \cdot \nabla_{\nabla h}(\gamma N)+\nabla^{2} \nabla_{\Delta h}(\gamma N) \\
= & N \partial_{h} \gamma-\partial_{x}\left(\gamma \frac{h_{x}}{N}\right)-\partial_{y}\left(\gamma \frac{h_{y}}{N}\right)-\partial_{x}\left(N \partial_{h_{x}} \gamma\right)-\partial_{y}\left(N \partial_{h_{y}} \gamma\right)+\varepsilon_{h o t} \\
= & N \partial_{h} \gamma-\frac{h_{x}^{2}+h_{y}^{2}}{N} \partial_{h} \gamma-\frac{h_{x} h_{x x}+h_{y} h_{x y}}{N} \partial_{h_{x}} \gamma-\frac{h_{y} h_{y y}+h_{x} h_{x y}}{N} \partial_{h_{y}} \gamma \\
& \quad-\gamma\left(\partial_{x}\left(\frac{h_{x}}{N}\right)+\partial_{y}\left(\frac{h_{y}}{N}\right)\right)-\partial_{x}\left(N \partial_{h_{x}} \gamma\right)-\partial_{y}\left(N \partial_{h_{y}} \gamma\right)+\varepsilon_{h o t} \\
= & \left(\partial_{h} \gamma\right) n_{3}-\gamma \kappa+\varepsilon_{\text {anis }}+\varepsilon_{\text {hot }}=\varepsilon_{\text {wet }}+\varepsilon_{\kappa}+\varepsilon_{\text {anis }}+\varepsilon_{\text {hot }} .
\end{aligned}
$$

As $\mathrm{SE}_{I V}$ is a generalization of the other energies we have established the results for $\mathrm{SE}_{I}-\mathrm{SE}_{I I I}$.

\section{Strong anisotropic surface energy}

Based on the analysis of the surface energy in the previous section we derive a new model for Stranski-Krastanov growth for surface energy type $\mathrm{SE}_{I V}$. It extends the model by Korzec and Evans [24] in that it accounts for a randomly perturbed flux and, more importantly, allows for large anisotropy coefficients. Here the surface energy transition between film and substrate is achieved by defining the overall surface energy density as

$$
\gamma=\frac{1}{2}\left(\gamma^{f}+\gamma^{s}\right)+\left(\gamma^{f}-\gamma^{s}\right) \frac{1}{\pi} \arctan \left(\frac{h}{b}\right)+\frac{1}{2} v \kappa^{2} .
$$

Such boundary-layer formulas have been used before [28, 35] as special case of a class of models with appropriate smooth transition functions of the surface energy of the substrate $\gamma^{s}$ to the surface energy of the film $\gamma^{f}$. This transition takes place on a small length scale $b$. As had been argued in [16], that near regions with sharp corners, where the curvature varies rapidly, the surface energy also depends on the surface curvature, leading to an additional contribution of $v \kappa^{2} / 2$, where the constant $v$ is a measure of the corner energy. The expressions $\gamma^{f}$ and $\gamma^{s}$ have been defined as constants in earlier works, while we let $\gamma^{f}$ depend on the orientation of the film. In this way, preferred facets have a smaller value than the forbidden orientations. We let

$$
\gamma^{f}\left(h_{x}, h_{y}\right)=\gamma_{0}\left(1+w\left(h_{x}, h_{y}\right)\right) .
$$

The anisotropy will be written as a general polynomial in the slopes of the surface

$$
w\left(h_{x}, h_{y}\right)=\sum_{k=1}^{N} \sum_{j=0}^{k} g_{k j} h_{x}^{j} h_{y}^{k-j},
$$

see, e.g., [24]. The general form (3.3) allows for various anisotropies, however, we restrict the polynomials in that $\gamma^{f} \rightarrow \infty$ for $h_{x}, h_{y} \rightarrow \pm \infty$. In this way large gradients, which are not allowed 
to be preferred in any case when using a small slope approximation, are punished. This behavior is also visible in the Wulff shape for the surface energy density formula for a quadruple well that leads to faceted pyramidal quantum dots, used in [24]. Setting $N=4, g_{20}=g_{02}=-2 g, g_{40}=g_{04}=$ $g$ and $g_{k j}=0$ for all other index pairs $(k, j)$, then the formula reads

$$
w\left(h_{x}, h_{y}\right)=g\left(h_{x}^{4}+h_{y}^{4}-2\left(h_{x}^{2}+h_{y}^{2}\right)\right), \quad g>0 .
$$

The resulting surface energy density (3.2) for the film is in fact a quadruple-well that fulfills the symmetry properties (2.3), so that (2.1) is fulfilled with the normals of the surface as argument. By using an angle-slope relation similar as in Figure 1 (c) we obtain the equilibrium shapes by applying the Wulff construction in Figure 2 (compare Figure 1 (a) and (b)). In (a) we see that the equilibrium shapes have rounded corners at the regions of interest (left and right corners - the upper and lower ones result from $h_{x} \rightarrow \pm \infty$ ). In (b) the anisotropy strength is increased from $g=0.15$ to $g=0.3$ and the previously smoothed out corners are now real kinks in the equilibrium shape, as is the case for the corresponding polar plots from Figure 1.

Furthermore, we include an atomic flux $f^{a}=\left(f_{1}, f_{2}, f_{3}\right)$ in the model. We note that the natural fluctuations that occur during deposition can be described via a Gaussian noise that perturbs a vertically impinging flux. Neglecting evaporation, the atoms are oriented downwards, $f_{3}<0$, the variations in $x$ and $y$ directions have the same probability and the flux at one space point at different time points is not correlated. If we denote the strength of the flux $f^{a}$ by the prescribed flux rate $F$, one can, out of these properties, deduce the expression

$$
f^{a}=-\frac{w}{|w|} F \quad \text { with } \quad w=\left(r\left(0, \sigma_{1}\right), r\left(0, \sigma_{1}\right), r\left(1, \sigma_{2}\right)\right)
$$

where $r(\tilde{\mu}, \tilde{\sigma})$ denotes a Gaussian random number with expectation $\tilde{\mu}$ and standard deviation $\tilde{\sigma}$. We use the expectations 0,0 and 1 and that standard deviations in the $z$ direction $\sigma_{1}$ may be different than for both horizontal directions, $\sigma_{2}$. The exact values do not have a significant influence on the surface evolution in the simulations. For simplicity we use the abbreviations $r_{1}=r\left(0, \sigma_{1}\right), r_{2}=$ $r\left(0, \sigma_{1}\right)$ and $r_{3}=r\left(1, \sigma_{2}\right)$. For the governing equations we obtain

$$
\begin{aligned}
h_{t} & =\frac{F}{|w|}\left(-r_{1} h_{x}-r_{2} h_{y}+r_{3}\right)+\sqrt{1+|\nabla h|^{2}} \nabla_{s}^{2} \mu, \quad(x, y, t) \in[0, L]^{2} \times[0, T], \\
\mu & =\varepsilon_{\text {sed }}+\varepsilon_{\kappa}+\varepsilon_{\text {anis }}+\varepsilon_{w e t}+\varepsilon_{h o t}, \\
\varepsilon_{\text {sed }} & =\left.\frac{1}{2} \sum_{i, j=1}^{3} \sigma_{i j} \epsilon_{i j}\right|_{z=h}, \\
\varepsilon_{\kappa} & =-\gamma \kappa, \\
\varepsilon_{\text {anis }} & =-2\left(\frac{h_{x} h_{x x}+h_{y} h_{x y}}{N} \partial_{h_{x}} \gamma+\frac{h_{y} h_{y y}+h_{x} h_{x y}}{N} \partial_{h_{y}} \gamma\right)-N \partial_{x} \partial_{h_{x}} \gamma-N \partial_{y} \partial_{h_{y}} \gamma, \\
\varepsilon_{w e t} & =\left(\partial_{h} \gamma\right) / N, \\
\varepsilon_{h o t} & =\left[\partial_{x x} \partial_{h_{x x}}+\partial_{x x} \partial_{h_{y y}}+\partial_{y y} \partial_{h_{x x}}+\partial_{y y} \partial_{h_{y y}}\right](\gamma N), \\
\gamma & =\frac{1}{2}\left(\gamma^{f}\left(h_{x}, h_{y}\right)+\gamma^{s}\right)+\left(\gamma^{f}\left(h_{x}, h_{y}\right)-\gamma^{s}\right) \frac{1}{\pi} \arctan \left(\frac{h}{b}\right)+\frac{1}{2} v \kappa^{2}, \\
\gamma^{f} & =\gamma_{0}\left(1+\sum_{k=2}^{N} \sum_{j=0}^{k} g_{k j} h_{x}^{j} h_{y}^{k-j}\right),
\end{aligned}
$$




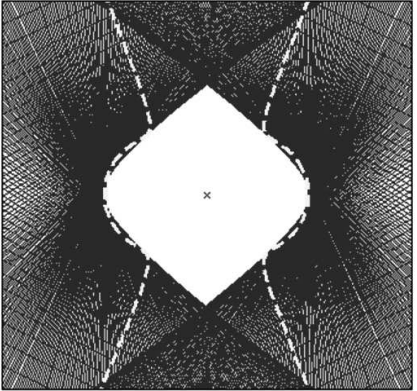

(a)

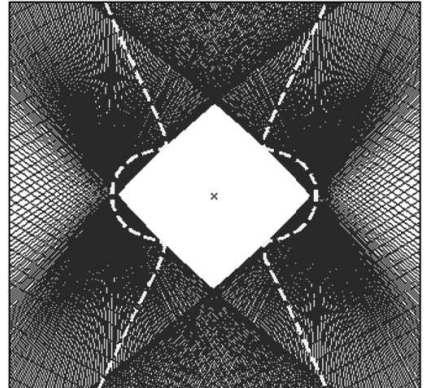

(b)

FIG. 2. Wulff shape constructions based on the anisotropy formula (3.4) in one dimension for two different anisotropy strengths. The dashed lines denote the polar plot of the surface energy density. (a) Weak anisotropy, $g<0.25$ leading to rounded shapes near $h_{x}=0$; (b) strong anisotropy, $g>0.25$ leading to corners in the equilibrium shapes.

where we note that the terms $\partial_{h_{x}} \gamma$ and $\partial_{h_{y}} \gamma$ appearing in the chemical potential (2.8) are given as derivatives of the anisotropy function $w$, for now left in its general form (3.3).

$$
\nabla_{\nabla h} \gamma=\left(\begin{array}{c}
\partial_{h_{x}} \\
\partial_{h_{y}}
\end{array}\right) \gamma=\gamma_{0} \Psi(b, h) \nabla_{\nabla h} w
$$

scaled by the transition function

$$
\Psi(b, h)=\frac{1}{2}+\frac{1}{\pi} \arctan (h / b) .
$$

The derivative is

$$
\nabla_{\nabla h} W=\left(\begin{array}{c}
\sum_{k=2}^{N} \sum_{j=1}^{k} g_{k j} j h_{x}^{j-1} h_{y}^{k-j} \\
\sum_{k=2}^{N} \sum_{j=0}^{k-1} g_{k j}(k-j) h_{x}^{j} h_{y}^{k-j-1}
\end{array}\right) .
$$

Furthermore note, that the elastic strain energy density $\varepsilon_{\text {sed }}$ for a dislocation free interface, the stresses in film and substrate are, result from the lattice mismatch $\epsilon$. The problem can be described in terms of linear elasticity, if the mismatch between the two materials is sufficiently small. In combination with Hooke's law and the fact that mechanical equilibrium is reached much faster than thermodynamic equilibrium, the elastic subproblem is given by the Navier-Cauchy equations

$$
\left(1-2 v^{f / s}\right) \nabla^{2} u^{f / s}+\nabla\left(\nabla \cdot u^{f / s}\right)=0,
$$

for the displacements $u^{f / s}$ in film and substrate, and the corresponding Poisson's ratio $v^{f / s}$. Additionally one treats the boundary conditions $\sigma \cdot n=0$ at $z=h, u_{i}^{s} \rightarrow 0$ for $z$ far below the substrate's surface, $u_{i}^{f}=u_{i}^{s}+\epsilon(x, y, 0)^{T}$ and continuity of the normal component of the stress at the substrate-film interface $z=0, \epsilon=\left(a^{f}-a^{s}\right) / a^{s}, a^{f}$ and $a^{s}$ being the lattice mismatch and the lattice spacings, respectively. Also periodicity in the horizontal directions can be assumed. It has been discussed in [44] how to obtain reduced expressions for the strain energy density out of this problem setting. 


\subsection{Thin film model}

A scaling under the thin-film assumption is [24, 44]

$$
\begin{gathered}
h=H_{0} H=\alpha L H, z=\alpha L Z,(x, y)=(L X, L Y), \\
t=\tau T, u_{i}(x, y, z)=L U_{i}(X, Y, Z), i=1,2,3,
\end{gathered}
$$

where $\alpha=H_{0} / L \ll 1$. Furthermore the transition thickness is chosen orders of magnitude smaller, it is scaled by $b=L \alpha^{3} \bar{b}$, so that the leading order of the wetting term $\varepsilon_{w e t}$ will be of the same order as the surface energy term $\varepsilon_{\kappa}$. To guarantee that the overall reduced model reflects the anisotropic behavior anticipated, orientation dependent variations have to be reflected in the leading order term of $\varepsilon_{\text {anis }}$. To capture the anisotropy in a reduced formula, all corresponding surface energy terms have to be incorporated in the evolution equation. Therefore these terms have to be of the same order, which is only the case when

$$
g_{k j}=G_{k j} \alpha^{-k+2}, \quad G_{k j}=\mathcal{O}(1) .
$$

In this way the slope polynomial becomes $W=\alpha^{2} W$ with $W$ defined by

$$
W\left(H_{X}, H_{Y}\right)=\sum_{k=1}^{N} \sum_{j=0}^{k} G_{k j} H_{X}^{j} H_{Y}^{k-j}
$$

From the nondimensionalization of the chemical potential

$$
\mu=\frac{\gamma_{0}}{L} \bar{\mu}, \quad \bar{\mu}=\bar{\varepsilon}_{s e d}+\bar{\varepsilon}_{\kappa}+\bar{\varepsilon}_{a n i s}+\bar{\varepsilon}_{w e t}+\bar{\varepsilon}_{h o t},
$$

and from the natural scalings

$$
\sigma_{i j}=\varepsilon_{\text {sed }}^{\text {base }} \bar{\sigma}_{i j}, \quad \kappa=\frac{1}{L} \bar{\kappa}, \quad \gamma=\gamma_{0} \bar{\gamma}, \quad \nabla_{s}^{2}=\frac{1}{L^{2}} \bar{\nabla}_{s}^{2}
$$

one can derive the characteristic length and time scales

$$
L=\frac{\gamma_{0}}{\varepsilon_{\text {sed }}^{\text {base }}} \quad \text { and } \quad \tau=\frac{L^{4}}{Ð \gamma_{0}} .
$$

Note, the nondimensionalization is achieved by using the expression $\varepsilon_{\text {sed }}^{\text {base }}=\epsilon^{2} E^{f} /\left(1-v^{f}\right)$ that is derived for a biaxially strained film on an undeformed substrate covering a half-space, where $E^{f}$ is Young's modulus and $v^{f}$ is Poisson's ratio of the film material.

The evolution equation in nondimensional form becomes

$$
H_{T}=\sqrt{1+\alpha^{2}\left(H_{X}^{2}+H_{Y}^{2}\right)} \frac{1}{\alpha}\left(\bar{\nabla}_{s}^{2}\left(\bar{\varepsilon}_{\text {sed }}+\bar{\varepsilon}_{\kappa}+\bar{\varepsilon}_{w e t}+\bar{\varepsilon}_{a n i s}+\bar{\varepsilon}_{h o t}\right)+\bar{f}\right),
$$


with the constituent potential terms

$$
\begin{aligned}
& \bar{\varepsilon}_{s e d}=\frac{1}{2} \sum_{i, j} \bar{\sigma}_{i j} \epsilon_{i j} \\
& \bar{\varepsilon}_{\kappa}=-\bar{\gamma} \bar{\kappa} \\
& \bar{\varepsilon}_{w e t}=\frac{\left(\gamma^{f}-\gamma^{s}\right) \alpha \bar{b}}{\gamma_{0} \pi\left(\alpha^{4} \bar{b}^{2}+H^{2}\right) \sqrt{1+\alpha^{2}|\nabla H|^{2}}}, \\
& \bar{\varepsilon}_{\text {anis }}=-2 \alpha \frac{\left(H_{X} H_{X X}+H_{X} H_{X Y}\right) \partial_{H_{X}} w+\left(H_{Y} H_{Y Y}+H_{Y} H_{X Y}\right) \partial_{H_{Y}} w}{\left(1+\alpha^{2}\left(H_{X}^{2}+H_{Y}^{2}\right)\right)^{1 / 2}} \\
& -\frac{1}{\alpha}\left(1+\alpha^{2}\left(H_{X}^{2}+H_{Y}^{2}\right)\right)^{1 / 2}\left(\partial_{X}\left[\Psi \partial_{H_{X}} w\right]+\partial_{Y}\left[\Psi \partial_{H_{Y}} w\right]\right) \\
& \bar{\varepsilon}_{h o t}=\frac{1}{\alpha} \nabla^{2}\left[\partial_{H_{X X}}+\partial_{H_{Y Y}}\right]\left(\frac{1}{2} \bar{\nu}^{2}\left(1+\alpha^{2}\left(H_{X}^{2}+H_{Y}^{2}\right)\right)^{1 / 2}\right),
\end{aligned}
$$

where in the last expression $\bar{v}=v /\left(\gamma_{0} L^{2}\right)$ and in the anisotropy term $\varepsilon_{\text {anis }}$ the transition function with rescaled arguments $\Psi=\Psi\left(L \alpha^{3} \bar{b}, H_{0} H\right)$ has been used. The flux term is

$$
\bar{f}=\tilde{F} \frac{-\alpha r_{1} H_{X}-\alpha r_{2} H_{Y}+r_{3}}{|w|}
$$

with the dimensionless deposition number

$$
\tilde{F}=\frac{F L^{3}}{D \gamma_{0}}
$$

Here, the randomly perturbed vector is $w=\left(r_{1}, r_{2}, r_{3}\right)^{T}$, defined as in equation (3.5). Now, all terms are expanded with respect to the small parameter $\alpha$. In particular, the leading order terms of the nondimensionalized chemical potential

$$
\bar{\mu}=\bar{\mu}^{(0)}+\alpha \bar{\mu}^{(1)}+\circlearrowleft\left(\alpha^{2}\right)
$$

and the flux

$$
\bar{f}=\bar{f}^{(0)}+\alpha \bar{f}^{(1)}
$$

will be determined. Derivative operators such as the nabla operator $\nabla=\left(\partial_{X}, \partial_{Y}\right)^{T}$ or the Laplacian $\nabla^{2}$ are now defined in the $(X, Y)$ variables. The surface Laplacian $\bar{\nabla}_{s}^{2}$ can be expanded to a standard Laplacian plus higher order perturbations, so that to order $\alpha$ the evolution equation can be written as

$$
H_{T}=\nabla^{2}\left(\frac{1}{\alpha} \bar{\mu}^{(0)}+\bar{\mu}^{(1)}\right)+\frac{1}{\alpha} \bar{f}^{(0)}+\bar{f}^{(1)} .
$$

The leading order terms of the chemical potential have already been already derived in [24, 44], giving the terms $\varepsilon_{\text {sed }}, \varepsilon_{\kappa}, \varepsilon_{w e t}, \varepsilon_{\text {anis }}$ : The strain energy density expansion is

$$
\bar{\varepsilon}_{\text {sed }}=\bar{\varepsilon}_{\text {sed }}^{(0)}+\alpha \bar{\varepsilon}_{\text {sed }}^{(1)}+\mathcal{O}\left(\alpha^{2}\right) .
$$

with

$$
\overline{\mathcal{E}}_{\text {sed }}^{(0)}=1 \quad \text { and } \quad \bar{\varepsilon}_{\text {sed }}^{(1)}=\mathcal{F}^{-1}[-\tilde{e} k \mathcal{F}[H]] .
$$


and the material constant

$$
\tilde{e}=\frac{2 \mu^{f}\left(1+v^{f}\right)\left(1-v^{s}\right)}{\left(1-v^{f}\right) \mu^{s}}
$$

which relates the elastic state of the substrate to the state of the film. Here $\mu^{f, s}$ is the shear modulus and $v^{f, s}$ is Poisson's ratio of the film and the substrate, respectively, and $k=\sqrt{k_{1}^{2}+k_{2}^{2}}$ is the length of the vector of two wave numbers $\left(k_{1}, k_{2}\right)$ that appear in the two dimensional Fourier transform $\mathcal{F}$ and its inverse $\mathfrak{F}^{-1}$.

From the constant part of the surface energy and the wetting layer one obtains the expansions

$$
\begin{gathered}
\bar{\varepsilon}_{\kappa}=\bar{\varepsilon}_{\kappa}^{(0)}+\alpha \bar{\varepsilon}_{\kappa}^{(1)}+\mathcal{O}\left(\alpha^{2}\right) \quad \text { with } \quad \bar{\varepsilon}_{\kappa}^{(0)}=0 \quad \text { and } \quad \bar{\varepsilon}_{\kappa}^{(1)}=-\nabla^{2} H \\
\bar{\varepsilon}_{w e t}=\bar{\varepsilon}_{w e t}^{(0)}+\alpha \bar{\varepsilon}_{w e t}^{(1)}+O\left(\alpha^{2}\right) \quad \text { with } \quad \bar{\varepsilon}_{w e t}^{(0)}=0 \quad \text { and } \quad \bar{\varepsilon}_{w e t}^{(1)}=-\frac{\tilde{\gamma}}{H^{2}},
\end{gathered}
$$

where $\tilde{\gamma}=\bar{b}\left(\gamma^{s}-\gamma_{0}\right) /\left(\gamma_{0} \pi\right)$. Usually $\tilde{\gamma}>0$, when a film covering the substrate is favorable.

The anisotropy results in

$$
\bar{\varepsilon}_{a n i s}=\bar{\varepsilon}_{a n i s}^{(0)}+\alpha \bar{\varepsilon}_{a n i s}^{(1)}+\mathcal{O}\left(\alpha^{2}\right)
$$

With the choice of the coefficients in $W$ as in (3.9) we have

$$
\bar{\gamma}=\gamma / \gamma_{0}=1+\alpha^{2} W\left(H_{X}, H_{Y}\right)+\frac{\alpha^{2}}{2} \bar{v}\left(\nabla^{2} H\right)^{2}+\mathcal{O}\left(\alpha^{3}\right),
$$

and $W$ is as in (3.10). Then the leading order expression is

$$
\bar{\varepsilon}_{\text {anis }}^{(1)}=-\frac{1}{\alpha^{2}} \nabla \cdot \nabla_{\nabla H} w\left(H_{X}, H_{Y}\right)=-\nabla \cdot \nabla_{\nabla H} W\left(H_{X}, H_{Y}\right) .
$$

For the higher order regularization term

$$
\bar{\varepsilon}_{h o t}=\bar{\varepsilon}_{h o t}^{(0)}+\alpha \bar{\varepsilon}_{h o t}^{(1)}+\mathcal{O}\left(\alpha^{2}\right)
$$

we set $\bar{v}=\alpha^{2} \tilde{v}$ to guarantee that the leading order term corresponds to the leading orders of the other surface energy terms. Then we obtain by expanding the terms in (3.14)

$$
\bar{\varepsilon}_{h o t}=\alpha \nabla^{2}\left[\partial_{H_{X X}}+\partial_{H_{Y Y}}\right]\left(\frac{1}{2} \tilde{v} \nabla^{2} H\left(1+\mathcal{O}\left(\alpha^{2}\right)\right)\right)=\tilde{v} \alpha \nabla^{4} H+\mathcal{O}\left(\alpha^{3}\right),
$$

and note the leading order terms

$$
\bar{\varepsilon}_{h o t}^{(0)}=0, \quad \bar{\varepsilon}_{h o t}^{(1)}=\tilde{v} \alpha \nabla^{4} H .
$$

Finally the deposition terms can be read off from

$$
\bar{f}=\tilde{F} \frac{-\alpha r_{1} H_{X}-\alpha r_{2} H_{Y}+r_{3}}{|w|}=\frac{\tilde{F} r_{3}}{|w|}-\alpha \frac{\tilde{F}}{|w|}\left(r_{1} H_{X}+r_{2} H_{Y}\right),
$$

giving

$$
\bar{f}^{(0)}=\frac{\tilde{F} r_{3}}{|w|} \text { and } \bar{f}^{(1)}=-\frac{\tilde{F}}{|w|}\left(r_{1} H_{X}+r_{2} H_{Y}\right)
$$


Recall that we choose the flux such that it models a perturbed beam of atoms which is oriented in the $(0,0,-1)$ direction.

Using $\nabla^{2} \mu^{(0)}=0$ the evolution equation (3.17) becomes

$$
H_{T}=\nabla^{2}\left(\bar{\varepsilon}_{\text {sed }}^{(1)}+\bar{\varepsilon}_{\kappa}^{(1)}+\bar{\varepsilon}_{w e t}^{(1)}+\bar{\varepsilon}_{\text {anis }}^{(1)}\right)+\bar{f}^{(0)} / \alpha+\bar{f}^{(1)} .
$$

Insertion of the derived terms (3.18), (3.20)-(3.24) into (3.25) results in the final evolution equation

$$
\begin{aligned}
& H_{T}=\nabla^{2}\left[\tilde{v} \nabla^{4} H-\nabla^{2} H-\tilde{\gamma} / H^{2}+\mathcal{F}^{-1}[-\tilde{e} k \mathcal{F}[H]]\right. \\
&\left.\quad-\nabla \cdot \nabla_{\nabla H} W\left(H_{X}, H_{Y}\right)\right]+\frac{\check{F} r_{3}}{|w|}-\frac{\tilde{F}}{|w|}\left(r_{1} H_{X}+r_{2} H_{Y}\right),
\end{aligned}
$$

where now

$$
\check{F}=\frac{F L^{4}}{Ð \gamma_{0} H_{0}} .
$$

This thin film model is stabilized by the linear sixth order term in comparison to the weak equation (where $\tilde{v}=0$ ). Furthermore, although the linear fourth order term has a stabilizing sign and may suggest that the regularization is not necessary, this is not true in general, as the overall stability depends on the polynomial $W$.

\section{Impact of strong anisotropy and deposition flux on faceting patterns}

We focus first on the linear stability analysis of flat films to infinitesimal perturbations of amplitude $\check{\delta} \ll 1$ for which a normal mode ansatz

$$
H=\bar{H}+\check{\delta} e^{\sigma t+i k_{1} X+i k_{2} Y},
$$

yields the dispersion relation for equation (3.26) for $F=0$

$$
\sigma=-\tilde{v} k^{6}-k^{4}+\tilde{e} k^{3}-\frac{2 \tilde{\gamma}}{\bar{H}^{3}} k^{2}-2 k^{2}\left(G_{20} k_{2}^{2}+G_{21} k_{1} k_{2}+G_{22} k_{1}^{2}\right) .
$$

For the case of materials with cubic symmetry the anisotropy constants in the $X$ and $Y$ directions are equal and that the crystal is aligned with those axes, $G_{20}=G_{22}=-2 G$ and $G_{21}=0$. With this choice of coefficients the relation simplifies to

$$
\sigma=-\tilde{v} k^{6}+(4 G-1) k^{4}+\tilde{e} k^{3}-\frac{2 \tilde{\gamma}}{\bar{H}^{3}} k^{2} .
$$

Here we see most clearly, that for a strong anisotropy coefficient $G$ the fourth order term becomes positive, leading to unstable behavior in case without the regularizing sixth order term. As noted before in [24] increased anisotropy leads to maxima at higher wave numbers in the dispersion relation and moreover above a critical value (here $G=1 / 4$ ) it has a completely destabilizing effect. As we observe now, the corner energy may drastically change the picture if allowed to increase. Figure 3 shows dispersion relations for two different anisotropy strengths and varying values of the regularization coefficient $\tilde{v}$. It is visible that as $\tilde{v} \rightarrow 0$ the dispersion curve tends to the weak case $\tilde{v}=0$. As $G>0.25$ this limit yields an ill-posed problem. 
While a recent study linear stability of thin solid films with strongly anisotropic surface energy and wetting interactions has been carried out by Khenner et al. [23], a more detailed discussion of the effects of strong anisotropy and corner regularization can best be found in Liu and Metiu [29], but also Cahn and Taylor [43]. Our results basically confirm their analysis and extension of Mullins' equation by including anisotropy and corner energy. Moreover, it is found that the coarsening mechanism will also be influenced by effects of anisotropy and corner energy. Next we will explore the nonlinear effect further by employing our numerical methods that enable us to follow the patterns over a long time range. We devote our attention to the influence of the coefficients $G$ and $\tilde{v}$ together with deposition flux $F$.

\subsection{Numerical simulations}

Our numerical simulations for equation (3.26) are based on a pseudospectral method, where we use a third order semi-implicit backward differentiation formula (SBDF) time-stepping procedure and an exponential time-differencing (ETD) method with a fourth order Runge-Kutta (RK4) timeintegration. Both are described in detail in the Appendix A.2, while here we present and discuss the results from our simulations. The linear stability analysis shows that a flat state above a certain critical thickness is unstable to perturbations (by choosing $\bar{H}$ small the unstable intervals seen in Figure 3 shrink until they vanish at a critical height dependent on the other parameters). Hence a random perturbation of a sufficiently large and constant initial condition shows the anticipated instability. First we made simulations for the case without deposition $(F=0)$ to see and understand the interplay between the anisotropy strength $G$ and the regularization coefficient $\tilde{v}$.

No deposition. In Figure 4 we see a typical evolution that is very similar to the case $\tilde{v}=0, G<$ $1 / 4$, discussed in [24], for which we use the same wetting and elastic parameters as in this reference, $\tilde{\gamma}=0.05, \tilde{e}=1.2778$, respectively, calculated for the $\mathrm{Ge} / \mathrm{Si}$ system, but a strong anisotropy $G=$ 0.5 and $\tilde{v}=0.2$. The flat film above the critical thickness develops a wrinkle pattern that evolves into faceted pyramids that are separated by a wetting layer. After long time of evolution as seen in Figure 5 that the pyramidal shape is very pronounced as faceting gets more preferred.

When $G$ is chosen larger, the anisotropy dominates the evolution. We observe in Figure 6 that a very strong anisotropy in fact stabilizes the faceted structures. Between $T=10$ and $T=480$ not
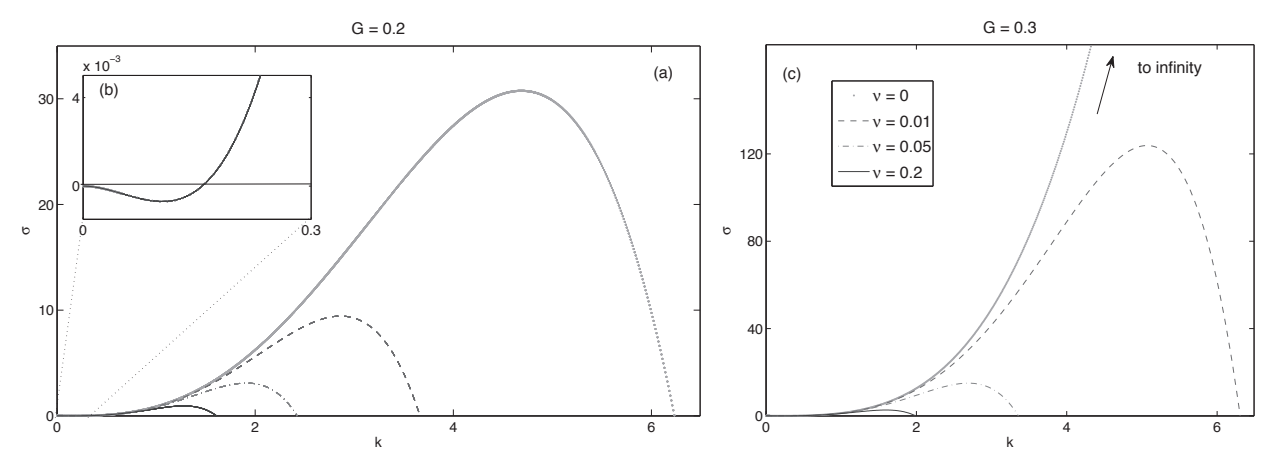

FIG. 3. Dispersion relation for the weak evolution equation $(v=0)$ and for the strong equation $(v>0)$ for an anisotropy value below the threshold $G=0.2<G_{c}=0.25$ and above, $G=0.3>G_{c}$. 

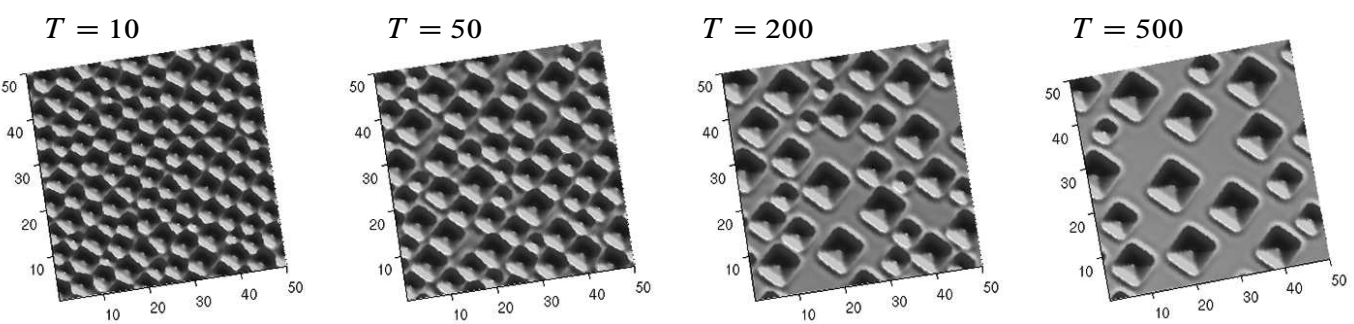

FIG. 4. Evolution based on equation (3.26) with $F=0, G=0.5, \tilde{v}=0.2$, starting with a flat surface above the critical height that is perturbed by white noise with a small amplitude.

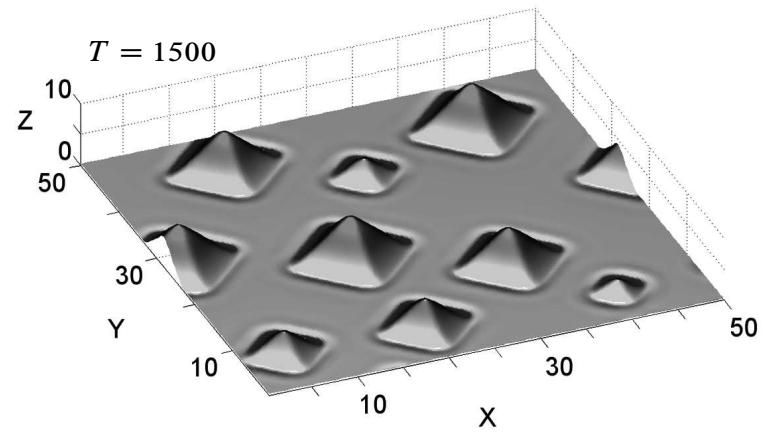

FIG. 5. Pyramidally shaped quantum dots after long-time evolution of equation (3.26) without deposition, $F=0$, simulated with the SBDF method

$$
T=10
$$

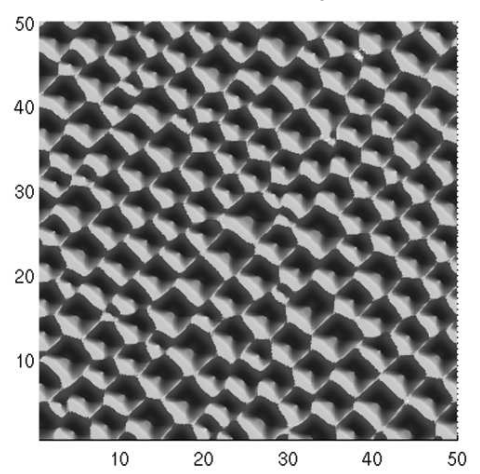

$$
T=480
$$

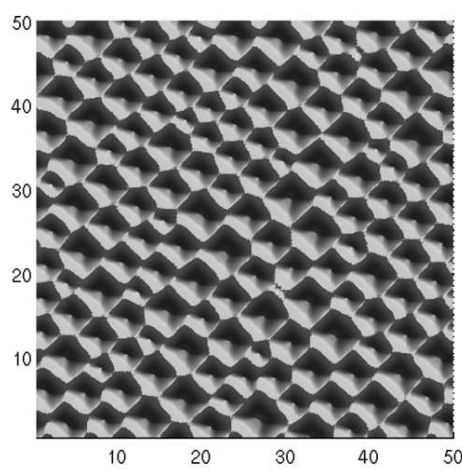

FIG. 6. Two time-points for the evolution with very strong anisotropy, $G=1$

much difference can be seen in the shapes and coarsening seems to come to a halt. This is in sharp contrast to the cases, where $\tilde{v}=0, G<0.25$, where coarsening always takes place.

To study the influence of the pair $(G, \tilde{v})$, which varies for different materials, on stationary states, we made computations on a small domain $[0,10]^{2}$ for different such pairs. Starting the simulations 

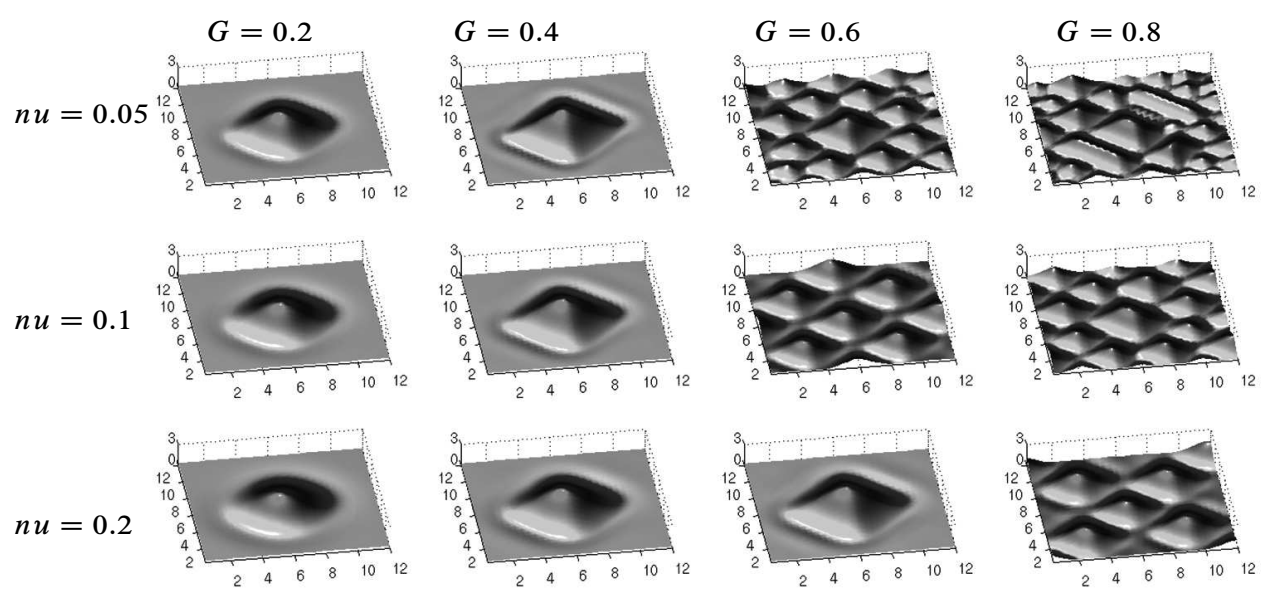

FIG. 7. Numerically calculated stationary states for different values of $\tilde{v}(=n u)$ and $G$.

with a Gaussian hump $h(x, y, 0)=2 \exp \left(-((x-5) / 2)^{2}-((y-5) / 2)^{2}\right)$ we continued the timestepping until a the relative change of two consecutive iterates falls below a small threshold. The results are plotted in Figure 7. While weak anisotropy with large regularization leads to a nearly isotropic dot, the other limit, strong anisotropy and small $\tilde{v}$, gives a completely faceted array of islands. The initial hump is decomposed smaller, faceted structures. With the regularizing term we indeed obtain, at least numerically, stabilized morphological structures, different from a single, stable pyramid, that is obtained in the weakly anisotropic case.

4.1.1 Deposition flux $\mathbf{F}>\mathbf{0}$.. When the flux of incoming atoms is nonzero, $F>0$, we observe coarsening as in Figure 8. Here we simulated coarsening process with three different values of $F$ and each column in the figure depicts the dimensionless time point, where the same amount of material has been deposited onto an initially flat surface. In all cases we see the typical coarsening events, smaller dots are eaten in expense of the bigger ones, however, with increased deposition rate, the number of islands remains higher, while the sizes of these dots are smaller. The additional income of atoms lets small pyramids survive longer. The high island density leads to more covering of the wetting region. This can also be read off from the coarsening diagram in Figure 9.

The diagram shows that the decrease in fact happens polynomially fast. The amount of deposited germanium atoms is plotted against the number of islands in a doubly logarithmic plot for three different flux rates. It is very well visible that more dots survive after the initial instability when the flux rate is higher.

\section{Summary and discussion}

In this study we have reviewed classes of surface energy formulations that have been proposed in models for epitaxial growth and developed a systematic framework to derive the surface energy for heteroepitaxial growth of a thin crystalline film. We presented a new model, where the surface energies also take account of a large anisotropy as well as the effect of wetting energy, elastic energy of the film and substrate and a randomly perturbed atomic deposition flux. We derived a 


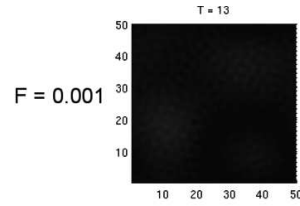

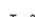
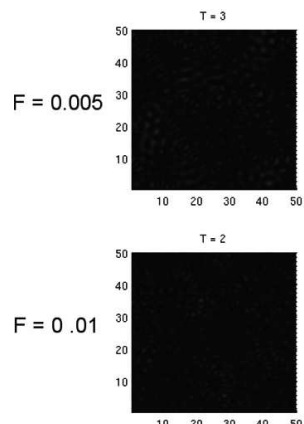

$T=36$

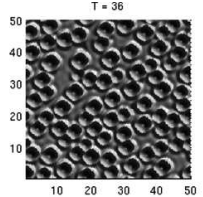

$T=7$

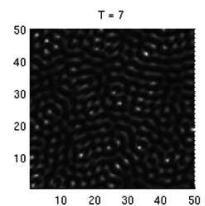

$T=4$

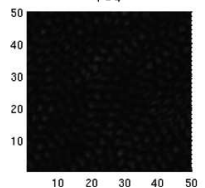

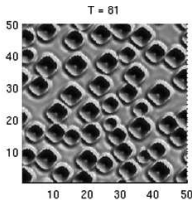

$T=10$
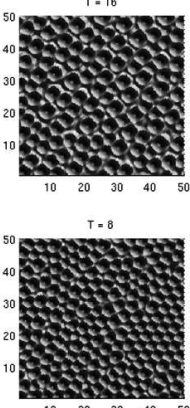

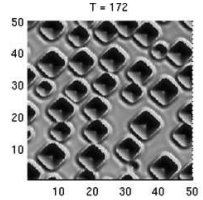

$r=34$

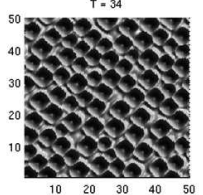

$T=17$

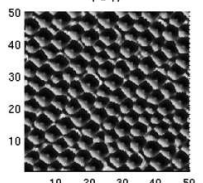

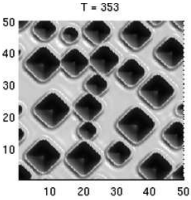

$T=7$

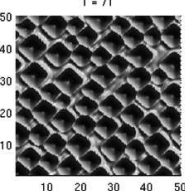

$T=36$

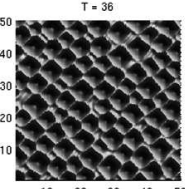

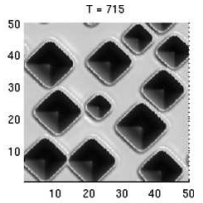

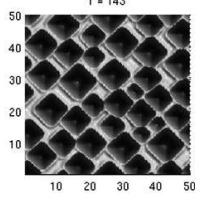

$T=72$

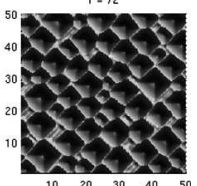

FIG. 8. Evolution of the thin films for $G=0.4, \tilde{v}=0.1$ for three different flux rates. Each picture in a column corresponds to the same amount of deposited film material and the flux is increased from one row to the next.

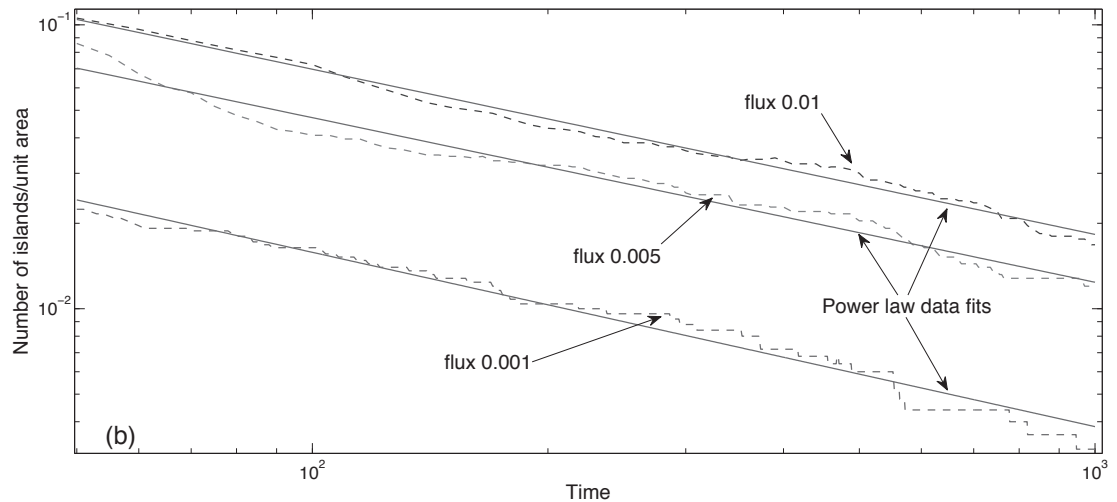

FIG. 9. Coarsening diagrams for $G=0.4, \tilde{v}=0.1$ and different flux rates. The number of atoms deposited is plotted against the normalized island density for three different fluxes.

corresponding thin film model and developed a numerical code that enables to capture the pattern evolution of the coarsening quantum dots.

We have shown that our pseudospectral method is sufficiently fast to simulate arrays of quantum dots for materials with strong anisotropy. The implicit-explicit schemes we tested had the drawback that high order schemes have unfavorable time-step restrictions, making simulations very slow. We found that the ETDRK4 method is well suited if one wants to obtain very accurate results on one hand, while relaxing the time-step restrictions. Optimized codes in terms of time-stepping and GPU utilization are the topic an ongoing study.

When the anisotropy parameters of the surface energy density are chosen very large, the dots are stabilized against coarsening. The energy wells of the preferred facets are so large, that this prevents 
the surface from fast ripening. Then the wetting effect becomes negligible and the evolution reminds more of perfectly faceted surfaces. We see from our numerical experiment on stationary states that dependent on $\gamma$ the qualitative and quantitative differences of the morphology are immense, ranging from isotropically shaped stationary states to small-scale faceted arrays. It hence is an important task to use a realistic surface energy formula with properly determined anisotropy and regularization coefficients.

The numerical results suggest also analytical studies on existence and investigations into the $\omega$ limit set, both are not known. Such results may depend strongly on the anisotropy and regularization parameters. The existence of stationary patterns of the nanostructures is in particular interesting for applications. Analytical results would be useful to confirm the numerical findings.

We believe that our model represents a realistic setting that can be used for comparison to experiments. Apart from investigating the impact of anisotropy on stability and coarsening behavior, it will be necessary to obtain knowledge about the strength of the corner energy. For material systems of large anisotropy (above the critical value of $\mathrm{G}$ ), assuming a slow coarsening, a measure of the pattern size that corresponds to the theoretical most preferred wavelength, would indirectly imply or at least narrow the range for the strength of the corner energy.

Acknowledgment MK and BW would like to acknowledge the financial support by the DFG Research Center MATHEON, project C10 and the photovoltaic competence center PVcomB.

\section{A. Appendix}

\section{A.1 Proofs for Section 2.1}

\section{A.1.1 Proof of Lemma 2.1.}

Proof. For the proof we need to show that the Householder matrices change the signs of the components of $n$, and fulfill one of the $3 !=6$ possible permutations.

Sign switch, $j \in\{1,2,3\}$ : With the Kronecker delta $\delta_{i j}$ we get

$$
H\left(a_{j}\right) n=\left(\begin{array}{ccc}
1-2 \delta_{1 j} & 0 & 0 \\
0 & 1-2 \delta_{2 j} & 0 \\
0 & 0 & 1-2 \delta_{3 j}
\end{array}\right)\left(\begin{array}{l}
n_{1} \\
n_{2} \\
n_{3}
\end{array}\right)=\left(\begin{array}{c}
\left(1-2 \delta_{1 j}\right) n_{1} \\
\left(1-2 \delta_{2 j}\right) n_{2} \\
\left(1-2 \delta_{3 j}\right) n_{3}
\end{array}\right)
$$

which is just one sign swith at position $j$ of the 3 -vector. The equality $\gamma(n)=\gamma\left(H\left(a_{j}\right) n\right)$ allows for repeated application to get several sign switches at once. Now we need to take care of the permutations.

Permutations: $j=4$ :

$$
H\left(a_{4}\right) n=I d-\left(\begin{array}{ccc}
1 & -1 & 0 \\
-1 & 1 & 0 \\
0 & 0 & 0
\end{array}\right)\left(\begin{array}{l}
n_{1} \\
n_{2} \\
n_{3}
\end{array}\right)=\left(\begin{array}{lll}
0 & 1 & 0 \\
1 & 0 & 0 \\
0 & 0 & 1
\end{array}\right)\left(\begin{array}{l}
n_{1} \\
n_{2} \\
n_{3}
\end{array}\right)=\left(\begin{array}{l}
n_{2} \\
n_{1} \\
n_{3}
\end{array}\right) .
$$

$j=5$ :

$$
H\left(a_{5}\right) n=I d-\left(\begin{array}{ccc}
1 & 0 & -1 \\
0 & 0 & 0 \\
-1 & 0 & 1
\end{array}\right)\left(\begin{array}{l}
n_{1} \\
n_{2} \\
n_{3}
\end{array}\right)=\left(\begin{array}{lll}
0 & 0 & 1 \\
0 & 1 & 0 \\
1 & 0 & 0
\end{array}\right)\left(\begin{array}{l}
n_{1} \\
n_{2} \\
n_{3}
\end{array}\right)=\left(\begin{array}{l}
n_{3} \\
n_{2} \\
n_{1}
\end{array}\right) .
$$

The concatenation of these Householder matrices yields all possible permutations. 


\section{A.1.2 Proof of Proposition 2.2.}

Proof. Any $n \in \mathbb{S}^{2}$ has a polar representation, $n=\left(n_{1}, n_{2}, n_{3}\right)^{T}=\left(\sin \left(\theta_{2}\right) \cos \left(\theta_{1}\right)\right.$, $\left.-\sin \left(\theta_{1}\right) \cos \left(\theta_{2}\right), \cos \left(\theta_{2}\right)\right)^{T}$. Then a sign switch of one of the angles or the addition or subtraction of $\pi / 2$ can be represented by two Givens rotations $R_{1}\left(\tilde{\theta}_{1}\right) R_{2}\left(\tilde{\theta}_{2}\right)$, where

$$
R_{1}\left(\tilde{\theta}_{1}\right)=\left(\begin{array}{ccc}
c\left(\tilde{\theta}_{1}\right) & s\left(\tilde{\theta}_{1}\right) & 0 \\
-s\left(\tilde{\theta}_{1}\right) & c\left(\tilde{\theta}_{1}\right) & 0 \\
0 & 0 & 1
\end{array}\right) \quad \text { and } \quad R_{2}\left(\tilde{\theta}_{1}\right)=\left(\begin{array}{ccc}
c\left(\tilde{\theta}_{2}\right) & 0 & s\left(\tilde{\theta}_{2}\right) \\
0 & 1 & 0 \\
-s\left(\tilde{\theta}_{2}\right) & 0 & c\left(\tilde{\theta}_{2}\right)
\end{array}\right)
$$

and we abbreviate $c=\cos$ and $s=\sin$. However, we have seen that the symmetry (2.1) can be expressed in terms of Householder transformations. As $\operatorname{det}\left(H\left(a_{j}\right)\right)=-1$ there cannot exist any rotation giving the same result, since $\operatorname{det}\left(R_{1} R_{2}\right)=\operatorname{det}\left(R_{1}\right) \operatorname{det}\left(R_{2}\right)=1$.

Although the equivalence does not hold, we have an implication. As two reflections $H_{1}, H_{2}$ fulfill $\operatorname{det}\left(H_{1} H_{2}\right)=1$, the previous argument cannot be reversed.

For $\delta \in\{ \pm 1\}$ it is $s(\theta)=\delta s(\delta \theta)$ and $c(\theta)=c(\delta \theta)$, hence we get for any $\delta_{j} \in\{ \pm 1\}, j=1,2$,

$$
\begin{aligned}
\gamma_{2}\left(\theta_{1}, \theta_{2}\right) & =\gamma_{1}\left(n_{1}, n_{2}, n_{3}\right)=\gamma_{1}\left(\delta_{2} n_{1}, \delta_{1} n_{2}, n_{3}\right) \\
& =\gamma_{1}\left(\delta_{2} s\left(\theta_{2}\right) c\left(\theta_{1}\right),-\delta_{1} s\left(\theta_{1}\right) c\left(\theta_{2}\right), c\left(\theta_{2}\right)\right) \\
& =\gamma_{1}\left(s\left(\delta_{2} \theta_{2}\right) c\left(\delta_{1} \theta_{1}\right),-s\left(\delta_{1} \theta_{1}\right) c\left(\delta_{2} \theta_{2}\right), c\left(\delta_{2} \theta_{2}\right)\right)=\gamma_{2}\left(\delta_{1} \theta_{1}, \delta_{2} \theta_{2}\right) .
\end{aligned}
$$

Now we prove the $\pi / 2$ rotation invariance. We show only what happens when $\theta_{2} \rightarrow \theta_{2}+\pi / 2$, the other cases follow analogously. A $\pi / 2$ increase of $\theta_{2}$ means an additional Givens rotation for the normal $\left(n \rightarrow \tilde{n}=\left(n_{3}, n_{2}, n_{1}\right)^{T}\right)$, we get

$$
\begin{aligned}
\gamma_{2}\left(\theta_{1}, \theta_{2}\right) & =\gamma_{1}\left(\left(\begin{array}{l}
n_{1} \\
n_{2} \\
n_{3}
\end{array}\right)\right)=\gamma_{1}\left(\left(\begin{array}{c}
n_{3} \\
n_{2} \\
-n_{1}
\end{array}\right)\right)=\gamma_{1}\left(\left(\begin{array}{ccc}
0 & 0 & 1 \\
0 & 1 & 0 \\
-1 & 0 & 0
\end{array}\right) n\right) \\
& =\gamma_{1}\left(\left(\begin{array}{ccc}
c(\pi / 2) & 0 & s(\pi / 2) \\
0 & 1 & 0 \\
-s(\pi / 2) & 0 & c(\pi / 2)
\end{array}\right) n\right)=\gamma_{2}\left(\theta_{1}, \theta_{2}+\pi / 2\right) .
\end{aligned}
$$

\section{A.1.3 Proof of Proposition 2.3.}

Proof. Since the surface is smoothly parametrized, the third component of $n$ is always bigger than or equal to zero, $n_{3}(a, b) \geqslant 0, \forall(a, b) \in \Omega$, and hence $\delta_{3}=+1$ in (2.1). Furthermore the 3-permutation $\pi=(\pi(1), \pi(2), \pi(3))$ is only permitted if $n_{\pi(3)} \geqslant 0$. Let $\delta_{1}, \delta_{2} \in\{ \pm 1\}$ be arbitrary.

" $\Rightarrow$ " Let (2.1) hold and $\pi(n)=\left(n_{2}, n_{1}, n_{3}\right)$, then

$$
\begin{aligned}
& \gamma_{3}\left(h_{x}, h_{y}\right)=\gamma_{1} \circ n\left(h_{x}, h_{y}\right)=\gamma_{1}\left(n_{1}, n_{2}, n_{3}\right) \\
& \quad=\left\{\begin{array}{c}
\gamma_{1}\left(\delta_{1} n_{1}, \delta_{2} n_{2}, n_{3}\right)=\gamma_{1} \circ n\left(\delta_{1} h_{x}, \delta_{2} h_{y}\right)=\gamma_{3}\left(\delta_{1} h_{x}, \delta_{2} h_{y}\right) \\
\gamma_{1}\left(\pi\left(n_{1}, n_{2}, n_{3}\right)\right)=\gamma_{1}\left(n_{2}, n_{1}, n_{3}\right)=\gamma_{3}\left(h_{y}, h_{x}\right)
\end{array} .\right.
\end{aligned}
$$

" $\Leftarrow$ " Let (2.2) hold, then as before $\gamma_{1}\left(n_{1}, n_{2}, n_{3}\right)=\gamma_{3}\left(h_{x}, h_{y}\right)=\gamma_{3}\left(\delta_{1} h_{x}, \delta_{2} h_{y}\right)=$ $\gamma_{1}\left(\delta_{1} n_{1}, \delta_{2} n_{2}, n_{3}\right)$. There is nothing to show for the interchange of the first two normal components since one can directly calculate $\gamma_{1}\left(n_{1}, n_{2}, n_{3}\right)=\gamma_{3}\left(h_{y}, h_{x}\right)=\gamma_{1}\left(n_{2}, n_{1}, n_{3}\right)$. 
Let $n_{3}$ permute with one of the other components (consider $\pi(n)=\left(n_{3}, n_{2}, n_{1}\right)$, the other case can be treated analogously). Since $n_{1}>0$, there exist $(a, b) \in \mathbb{R}^{2}$ such that $\left(n_{3}, n_{2}, n_{1}\right)=$ $(-a,-b, 1) / \sqrt{1+a^{2}+b^{2}}$. Since $\left(n_{1}, n_{2}, n_{3}\right)=\left(-h_{x},-h_{y}, 1\right) / N$, one obtains the identity

$$
\left(1,-h_{y},-h_{x}\right) / \sqrt{1+h_{x}^{2}+h_{y}^{2}}=(-a,-b, 1) / \sqrt{1+a^{2}+b^{2}},
$$

which gives $b=-h_{y} / h_{x}$ and $a=1 / h_{x}$. Hence

$$
\gamma_{1}\left(\pi\left(n_{1}, n_{2}, n_{3}\right)\right)=\gamma_{1}\left(n_{3}, n_{2}, n_{1}\right)=\gamma_{3}(a, b)=\gamma_{1}\left(n_{1}, n_{2}, n_{3}\right)
$$

because of (A.2).

\section{A.2 The numerical schemes}

We solved evolution equation (3.26) for the case without deposition, $F=0$, with a pseudospectral method, by transforming the two spatial variables into Fourier space and using a time-stepping scheme discussed by Ascher, Ruuth and Wetton [2]. For the case with deposition, $F>0$, we used an exponential time differencing (ETD) type method introduced by Cox and Matthews [11] and improved by Kassam and Trefethen [22]. The first method is a third order semi-implicit backward differentiation (SBDF) scheme. For an equation

$$
u_{t}=\mathrm{L} u+\mathrm{N}(u)
$$

with a linear part $\mathrm{L} u$ and the nonlinearity $\mathrm{N}(u)$, the update for the $n+1$ st time step is defined by

$$
\frac{11}{6} u^{n+1}-3 u^{n}+\frac{3}{2} u^{n-1}-\frac{1}{3} u^{n-2}=d t\left[3 \mathrm{~N}\left(u^{n}\right)-3 \mathrm{~N}\left(u^{n-1}\right)+\mathrm{N}\left(u^{n-2}\right)+\mathrm{L} u^{n+1}\right],
$$

where one calculates the new iterate $u^{n+1}$ from the three previous steps $u^{j}, j=n, n-1, n-2$ and $d t$ is a fixed stepsize. It can be applied to our evolution equation, since in Fourier space, discretized, with the DFT $u=F(H)$ it becomes just (A.3) with the diagonal linearity

$$
\mathrm{L}=\operatorname{diag}\left(\left\{-\tilde{v} k^{6}-k^{4}+\bar{e} k^{3}\right\}_{\left(k_{1}, k_{2}\right) \in \mathcal{K}}\right)
$$

where $\mathcal{K} \subset \mathbb{R}^{2}$ is a set of suitable wavenumber pairs and $k=\sqrt{k_{1}^{2}+k_{2}^{2}}$. The nonlinear part reads

$$
\left(\mathrm{N}(u)\left(k_{1}, k_{2}\right)\right)_{\left(k_{1}, k_{2}\right) \in \mathcal{K}}=\left(k^{2}\left(i k_{1} \hat{\Psi}_{1}\left(k_{1}, k_{2}\right)+i k_{2} \hat{\Psi}_{2}\left(k_{1}, k_{2}\right)+\tilde{\gamma} \hat{\Psi}_{3}\left(k_{1}, k_{2}\right)\right)\right)_{\left(k_{1}, k_{2}\right) \in \mathcal{K}} .
$$

Here

$$
\begin{aligned}
& \hat{\Psi}_{j}=\mathcal{F}\left[G\left(\left[\mathcal{F}^{-1}\left[i\left(k_{j} u\left(k_{1}, k_{2}\right)\right)_{\left(k_{1}, k_{2}\right) \in \mathcal{K}}\right]\right]^{3}-i\left(k_{j} u\left(k_{1}, k_{2}\right)\right)_{\left(k_{1}, k_{2}\right) \in \mathcal{K}}\right)\right], \quad j=1,2 \\
& \hat{\Psi}_{3}=\mathcal{F}\left[\frac{1}{\left(\mathcal{F}^{-1}[u]\right)^{2}}\right]
\end{aligned}
$$

for the special case of the quadruple-well anisotropy (3.4). It could be replaced by other anisotropic expressions to model different materials. The shown figures were calculated with $128^{2}$ wave number pairs and time-step size $d t=1 e-4$. 
For the stationary islands discussed in the last Section (i.e. Figure 7) we evolved the surface until the updated iterate, the shape $h_{+}$, fulfills

$$
\left\|h_{+}-h\right\|_{2} /\|h\|_{2}<d t \epsilon
$$

for some small tolerance $\epsilon$.

For the case with deposition we used the same linearity (A.4) and added the flux terms to the nonlinearity. We could have defined the deposition as part of the linearity, however, this would not work well with the ETD method that we applied. This update writes

$$
u_{n+1}=e^{\mathrm{L} d t} u_{n}+\alpha_{1} \mathrm{~N}\left(u_{n}, t_{n}\right)+\alpha_{2}\left(\mathrm{~N}\left(a_{n}, t_{n}+d t / 2\right)+\mathrm{N}\left(b_{n}, t_{n}+d t / 2\right)\right)+\alpha_{3} \mathrm{~N}\left(c_{n}, t_{n}+d t\right)
$$

with the variable coefficients

$$
\begin{aligned}
& a_{n}=e^{\mathrm{L} d t / 2} u_{n}+\mathrm{L}^{-1}\left(e^{\mathrm{L} d t / 2}-I\right) \mathrm{N}\left(u_{n}, t_{n}\right) \\
& b_{n}=e^{\mathrm{L} d t / 2} u_{n}+\mathrm{L}^{-1}\left(e^{\mathrm{L} d t / 2}-I\right) \mathrm{N}\left(a_{n}, t_{n}+d t / 2\right) \\
& c_{n}=e^{\mathrm{L} d t / 2} a_{n}+\mathrm{L}^{-1}\left(e^{\mathrm{L} d t / 2}-I\right)\left(2 \mathrm{~N}\left(b_{n}, t_{n}+d t / 2\right)-\mathrm{N}\left(u_{n}, t_{n}\right)\right)
\end{aligned}
$$

and the cancellation error prone coefficients

$$
\begin{aligned}
& \alpha_{1}=d t^{-2} \mathrm{~L}^{-3}\left[-4-\mathrm{L} d t+e^{\mathrm{L} d t}\left(4-3 \mathrm{~L} d t+(\mathrm{L} d t)^{2}\right)\right] \\
& \alpha_{2}=2 d t^{-2} \mathrm{~L}^{-3}\left[2+\mathrm{L} d t+e^{\mathrm{L} d t}(-2+\mathrm{L} d t)\right] \\
& \alpha_{3}=d t^{-2} \mathrm{~L}^{-3}\left[-4-3 \mathrm{~L} d t-(\mathrm{L} d t)^{2}+e^{\mathrm{L} d t}(4-\mathrm{L} d t)\right]
\end{aligned}
$$

that we evaluate with help of a Cauchy integral as it has been proposed in [22]. If the linearity would be time-dependent (by adding deposition), the coefficients would have to be calculated in each time step, making the calculations costly. The method seems more efficient than high order SBDF schemes. Additionally the ETD method has a better time-step restriction.

\section{REFERENCES}

1. AQUA, J.-N., Frisch, T., \& VERGA, A., Nonlinear evolution of a morphological instability in a strained epitaxial film, Phys. Rev. B 76 (2007), 165319.

2. Ascher, U. M., RuUth, S. J., \& Wetton, B., Implicit-explicit methods for time-dependent partial differential equations, SIAM J. Numer. Anal. 32 (1995), 797-823. Zb10841. 65081 MR1335656

3. Brown, G. F., \& WU, J., Third generation photovoltaics, Laser \& Photon 3 (2009), 394-405.

4. Burger, M., Hausser, F., StÖcker, C., \& Voigt, A., A level set approach to anisotropic flows with curvature regularization, J. Comput. Phys. 225 (2007), 183-205. Zbl1201. 82049 MR2346676

5. Caha, O., Holý, V., \& Bassler, K. E., Nonlinear Evolution of Surface Morphology in InAs/AlAs Superlattices via Surface Diffusion, Phys. Rev. Lett. 96 (2006), 136102.

6. Chen, W.-Y., Chang, W. H., Chou, A. T., Hsu, T. M., Chen, P. S., \& Z. Pei, L.-S. L., Optical properties of stacked $\mathrm{Ge} / \mathrm{Si}$ quantum dots with different spacer thickness grown by chemical vapor deposition, Appl. Surf. Sci. 224 (2004), 148-151.

7. CHIU, C.-H., The self-assembly of uniform heteroepitaxial islands, Appl. Phys. Lett. 75 (1999), 34733475 . 
8. CHIU, C.-H., Stable and uniform arrays of self-assembled nanocrystalline islands, Phys. Rev. B 69 (2004), 165413.

9. Chiu, C.-H., \& Huang, Z., Common features of nanostructure formation induced by the surface undulation on the Stranski-Krastanow systems, Appl. Phys. Lett. 89 (2006), 171904.

10. Chiu, C.-H., \& HUANG, Z., Numerical simulation for the formation of nanostructures on the StranskiKrastanow systems by surface undulation, J. Appl. Phys. 101 (2007), 113540.

11. Cox, S. M., \& Matthews, P. C., Exponential Time Differencing for Stiff Systems, J. Comp. Phys. 176 (2002), 430-455. Zbl1005.65069 MR1894772

12. Drucker, J., Self-Assembling Ge(Si)/Si(001) Quantum Dots, IEEE J. Quantum Electronics 38 (2002), 975-987.

13. EisenberG, H. R., \& KAndel, D., Wetting Layer Thickness and Early Evolution of Epitaxially Strained Thin Films, Phys. Rev. Lett. 85 (2000), 1286-1289.

14. Eisenberg, H. R., \& Kandel, D., Origin and properties of the wetting layer and early evolution of epitaxially strained thin films, Phys. Rev. B 66 (2002), 155429.

15. Eisenberg, H. R., \& KAndel, D., Formation, ripening, and stability of epitaxially strained island arrays, Phys. Rev. B 71 (2005), 115423.

16. Golovin, A. A., Davis, S. H., \& Nepomnyashchy, A. A., A convective Cahn-Hilliard model for the formation of facets and corners in crystal growth, Phys. D 122 (1998), 202-230. Zb10952.74050 MR1650139

17. Golovin, A. A., Davis, S. H., \& Voorhees, P. W., Self-organization of quantum dots in epitaxially strained solid films, Phys. Rev. E 68 (2003), 056203.

18. Golovin, A. A., Levine, M. S., Savina, T. V., \& DaVis, S. H., Faceting instability in the presence of wetting interactions: A mechanism for the formation of quantum dots, Phys. Rev. B 70 (2004), 235342.

19. Herring, C., Some theorems on the free energies of crystal surfaces, Phys. Rev. 82 (1951), 87-93. Zbl0042.23201

20. Johnston, K. W., Pattantyus-Abraham, A. G., Clifford, J. P., Myrskog, S. H., Macneil, D. D., LEVINA, L., \& SARGENT, E. H., Schottky-quantum dot photovoltaics for efficient infrared power conversion, App. Phys. Lett. 92 (2008), 151115.

21. Kamat, P. V., Quantum Dot Solar Cells. Semiconductor Nanocrystals as Light Harvester, J. Phys. Chem. C 112 (2008), 18737-18753.

22. Kassam, A.-K., \& Trefethen, L. N., Fourth-order time-stepping for stiff PDEs, SIAM J. Sci. Comput. 26 (2005), 1214-1233. Zb11077.65105 MR2143482

23. Khenner, M., Tekalign, W. T., \& Levine, M. S., Stability of a strongly anisotropic thin epitaxial film in a wetting interaction with elastic substrate, EPL 93 (2011).

24. Korzec, M. D., \& Evans, P. L., From bell shapes to pyramids: A reduced continuum model for selfassembled quantum dot growth, Phys. D 239 (2010), 465-474. Zbl1186. 82081 MR2593041

25. Korzec, M. D., Evans, P. L., MÜNCh, A., \& WAgner, B., Stationary solutions of driven fourthand sixth-order Cahn-Hilliard type equations, SIAM J. Appl. Math. 69 (2008), 348-374. Zbl1171. 34037 MR2452863

26. Korzec, M. D., NAYAR, P., \& RybKa, P., Global weak solutions to a sixth order Cahn-Hilliard type equation, to appear in SIAM J. Math. Anal. (2012).

27. KorZec, M. D., \& RYB KA, P., On a higher order convective Cahn-Hilliard type equation, SIAM J. Appl. Math. 72 (2012), 1343-1360.

28. Kutka, R. V., \& Freund, L. B., Minimum energy configuration of epitaxial material clusters on a lattice-mismatched substrate, J. Mech. Phys. Solids 45 (1997).

29. LiU, F., \& Metiu, H., Dynamics of phase separation of crystal surfaces, Phys. Rev. B 48 (1993), 58085817. 
30. McFadden, G. B., Coriell, S. R., \& SEKERKA, R. F., Effect of surface tension anisotropy on cellular morphologies, J. Crystal Growth 91 (1988), 180-198.

31. Mullins, W. W., Theory of Thermal Grooving, J. Appl. Phys. 28 (1957), 333-339.

32. PANG, Y., \& HuANG, R., Nonlinear effect of stress and wetting on surface evolution of epitaxial thin films, Phys. Rev. B 74 (2006), 075413.

33. PANG, Y., \& HUANG, R., Effect of elastic anisotropy on surface pattern evolution of epitaxial thin films, Int. J. Sol. Struct. 46 (2009), 2822-2833. Zbl1167.74498

34. Savina, T. V., Golovin, A. A., Davis, S. H., Nepomnyashchy, A. A., \& Voorhees, P. W., Faceting of a growing crystal surface by surface diffusion, Phys. Rev. E 67 (2003), 021606.

35. SPEnCER, B. J., Asymptotic derivation of the glued-wetting-layer model and contact-angle condition for Stranski-Krastanow islands, Phys. Rev. B 59 (1999), 2011-2017.

36. Spencer, B. J., Asymptotic solutions for the equilibrium crystal shape with small corner energy regularization, Phys. Rev. E 69 (2004), 011603.

37. Spencer, B. J., Davis, S. H., \& Voorhees, P. W., Morphological instability in epitaxially strained dislocation-free solid films: Nonlinear evolution, Phys. Rev. B 47 (1993), 9760-9777.

38. Spencer, B. J., \& Tersoff, J., Stresses and first-order dislocation energetics in equilibrium StranskiKrastanow islands, Phys. Rev. B $6 \mathbf{3}$ (2001), 205424.

39. Spencer, B. J., Voorhees, P. W., \& Davis, S. H., Morphological instability in epitaxially strained dislocation-free solid films, Phys. Rev. Lett. 67 (1991), 3696-3699.

40. Spencer, B. J., Voorhees, P. W., \& Davis, S. H., Morphological instability in epitaxially strained dislocation-free solid films: Linear stability theory, J. Appl. Phys. 73 (1993), 4955-4970.

41. Springholz, G., \& Holy, V., Stacking and Ordering in Self-Organized Quantum Dot Multilayer Structures, Lateral alignment of epitaxial quantum dots, ed. O. Schmidt (Springer Series on Nanoscience and Technology) (2008), 245-303.

42. Sutter, E., Sutter, P., \& Vescan, L., Organization of self-assembled quantum dots in SiGe/Si multilayers: effect of strain and substrate curvature, Mat. Sci. Eng. B89 (2002), 196-200.

43. TAYlor, J. E., \& CAHn, J. W., Diffuse interfaces with sharp corners and facets: Phase field models with strongly anisotropic surfaces, Physica D 112 (1998), 381-411. Zbl0930. 35201 MR1607466

44. Tekalign, W. T., \& Spencer, B. J., Evolution equation for a thin epitaxial film on a deformable substrate, J. Appl. Phys. 96 (2004), 5505-5512.

45. Tekalign, W. T., \& Spencer, B. J., Thin-film evolution equation for a strained solid film on a deformable substrate: Numerical steady states, J. Appl. Phys. 102 (2007), 073503.

46. WulfF, G., Zur Frage der Geschwindigkeit des Wachstums und der Auflösung der Krystallflächen, Zeitschrift f. Krystall. Mineral. 34 (1901), 449-530.

47. Xiang, Y., \& E, W., Nonlinear evolution equation for the stress-driven morphological instability, J. Appl. Phys. 91 (2002), 9414-9422.

48. Zhang, Y. W., Bower, A. F., \& LiU, P., Morphological evolution driven by strain induced surface diffusion, Thin Solid Films 424 (2003), 9-14.

49. Zhang, Y. W., Bower, A. F., XIA, L., \& Shin, C. F., Three dimensional finite element analysis of the evolution of voids and thin films by strain and electromigration induced surface diffusion, J. Mech. Phys. Solids 47 (1999), 173-199.

50. Zhang, Y. W., \& SRolovitz, D. J., Surface instability and evolution of nonlinear elastic heteroepitaxial thin-film structures, Phys. Rev. B 70 (2004), 041402. 